\title{
LA FILOSOFÍA DEL DERECHO DE ALEXANDRE KOJËVE*
}

\author{
Oriol Farrés Juste ** \\ Universidad Autónoma de Barcelona \\ Oriol.Farres@uab.es
}

RESUMEN. Este artículo es una presentación de la filosofía del derecho de Alexandre KOJÈVE contenida en su Esquisse d'une phénoménologie du droit (1981). La poca atención que dicha obra ha recibido es un vacío que debiera llenarse con una reflexión crítica de sus puntos fuertes. Entre ellos destaca una concepción de la justicia internacional que proyecta una luz muy singular sobre los actuales debates en torno a la globalización y el cosmopolitismo. A ojos de este autor, la ciudadanía es el elemento clave para aquilatar la expansión global de lo jurídico. En suma, KOJÈVE aparece como un valioso referente en la labor de pensar la contraposición entre lo jurídico y lo político que está en la base de toda filosofía del derecho, con la aspiración al logro de la justicia internacional y la paz mundial en el horizonte.

Palabras clave: fenomenología del derecho, cosmopolitismo, ciudadanía, derecho, política, paz.

\section{Alexandre Kojève's Philosophy of Law}

ABSTRACT. This article is a presentation of Alexandre KOJĖVE's philosophy of law, exposed in his Esquisse d'une phénoménologie du droit (1981). Little attention has been paid to this work. So there is a gap that has to be filled with a critical reflection of its strengths. Among them, undoubtedly, we count the fact that KOJĖVE is introducing a conception of international justice that casts a singular light on current debates about cosmopolitanism and globalization. According to this author, citizenship is the key element of the process of global expansion of the juridical sphere. In sum, KoJèVE's philosophy is useful to reflect upon the contrast between the juridical and the political, which is the basis for all philosophy of law, in order to achieve world peace and international justice.

Keywords: phenomenology of right, cosmopolitanism, citizenship, right, politics, peace.

\footnotetext{
* Fecha de recepción: 1 de junio de 2016. Fecha de aceptación: 13 de febrero de 2017.

Este artículo es resultado del proyecto de investigación FFI2015-64858-P, titulado: Justicia y democracia: hacia un nuevo modelo de solidaridad, y financiado por el Ministerio de Economía y Competitividad (España).

** Investigador Postdoctoral Docente. Departamento de Filosofía.
} 


\section{INTRODUCCIÓN}

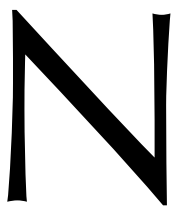

os proponemos abordar en lo que sigue las tesis más relevantes de la filosofía del derecho contenida en Esquisse d'une phénoménologie du droit (1981) de Alexandre KoJÈVE, autor más conocido por su Introduction à la lecture de Hegel (1968). Esta última es una recopilación, llevada a cabo por Raymond QUENEAU, de los apuntes correspondientes a las clases dictadas por KoJÈVE en l'École Pratique des Hautes Études de París, durante el que fue un celebérrimo seminario que marcó generaciones enteras en Francia y en el mundo entero por lo que respecta a la interpretación de la hegeliana Fenomenología del Espíritu. A raíz de ello y tras ingresar en la administración De Gaulle, KojèvE se convirtió en una enigmática eminencia gris que influyó sin ostentación en autores de la talla de Leo STRAUSS ${ }^{1}$, Georges BATAILlE o Jacques LACAN. La teoría del fin de la historia relanzada más tarde por Francis FuKUYAMA ${ }^{2}$ representó, sin lugar a dudas, la puesta al día de KOJÈvE en los debates políticos posteriores al término de la guerra fría y la implosión de la Unión Soviética. Sin embargo, aunque menos difundido más importante resulta, según acreditadas voces, su Esquisse d'une phénoménologie du droit $^{3}$. En este libro argumenta KOJÈvE sobre la plausibilidad del «Estado universal y homogéneo», que sería su propia versión acerca de una integración jurídica mundial de la que se seguiría la imposibilidad tanto de las guerras como de las revoluciones, de tal modo que el proyecto kantiano de la paz perpetua recibiría en dicha obra una validación no menor in bipothesi que in thesi $i^{4}$.

En el fondo se trata, en Esquisse d'une phénoménologie du droit, de desarrollar los temas del derecho, la justicia y el Estado universal y homogéneo que se encuentran ya en la Introduction à la lecture de Hegel, si bien todavía demasiado sujetos a la interpretación de HEGEL. En cambio, en el Esquisse el autor se muestra «satisfecho» con los resultados obtenidos hablando ya en primera persona, esto es, sin limitarse a glosar a Hegel (AufFret, 1990: 280). Enuncia además una serie de problemas y consideraciones muy pertinentes en el ámbito de la filosofía del derecho por sus consecuencias políticas y filosóficas de primer orden. Aun así, sorprendentemente ha sido muy escasa la atención que se le ha prestado si se exceptúa su reciente interpretación por François TERRÉ, Bryan-Paul Frost y Robert Howse. Pero aún queda mucho por extraer de dicha obra. Por lo pronto, en este artículo vamos a trasladar sus análisis, en la medi-

1 Especialmente importante es el debate entre KojÈve y Strauss en L. STRAuss, On Tyranny, Chicago, Chicago University Press, 2000 (hay traducción castellana: Sobre la tiranía, Madrid, Ediciones Encuentro, 2005).

2 F. FunuYama, The End of History and the Last Man, New York, Free Press, 1992 (hay traducción castellana: El fin de la Historia y el último bombre, Barcelona: Planeta, 1992).

3 P. Anderson, «The Ends of History», en A Zone of Engagement, New York, Verso, 1992, 320-321, especialmente nota 102 .

4 «Los Estados con relaciones recíprocas entre sí no tienen otro medio, según la razón, para salir de la situación sin leyes, que conduce a la guerra, que el de consentir leyes públicas coactivas, de la misma manera que los individuos entregan su libertad salvaje (sin leyes), y formar un Estado de pueblos (civitas gentium) que (siempre, por supuesto, en aumento) abarcaría finalmente a todos los pueblos de la tierra. Pero si por su idea del derecho de gentes no quieren esta solución, con lo que resulta que lo que es correcto in thesi lo rechazan in hipothesi, en este caso, el raudal de los instintos de injusticia y enemistad solo podrá ser detenido, en vez de por la idea positiva de una república mundial, por el sucedáneo negativo de una federación permanente y en continua expansión, si bien con la amenaza constante de que aquellos instintos estallen» (KANT, 2003: 25-26). 
da de lo posible, al problemático contexto actual de la globalización y las relaciones internacionales; es decir, trataremos de exponer el planteamiento kojeviano de la integración jurídica mundial como realización paulatina de la idea de justicia y clausura de su evolución histórica. Cabe reconocer que la obra se ha granjeado escasa atención incluso en Francia; existe, empero, una traducción al inglés ${ }^{5}$. Con nuestra presentación nos gustaría suplir un tanto la ausencia de su traducción al castellano, cuando menos hasta que esta se produzca, y en el mejor de los casos, como un incentivo para ello.

A pesar del título, Esquisse, el autor va más allá de un mero bosquejo de la cuestión y ofrece un desarrollo sistemático en tres secciones: el derecho como tal, su origen y evolución, y por último, el sistema del derecho; 588 páginas en total de la edición francesa (1981). KoJĖvE parte de una fenomenología del derecho, esto es, de una descripción de las condiciones a partir de las cuales tiene o no sentido hablar de «derecho». Como primer elemento destaca el trasfondo social de toda relación jurídica. En este contexto está absolutamente injustificado hablar con propiedad de «derechos individuales». La relación jurídica siempre es, por un lado, una relación plural e implica, por el otro, al conjunto de una sociedad; dicho de otro modo, los derechos son siempre colectivos y su razón de ser está en la interacción de los individuos, nunca en una propiedad individualizada (KoJÈve, 1981: 112-15, 155-56 y 367 ).

En este contexto «fenomenología» significa descripción a partir de características visibles o, como establece el propio autor, en términos de un paradigma bebaviorista. Se basará, pues, en la observación: «Para llegar a tal definición "behaviorista" del derecho, veamos cómo actúan los hombres cuando se hallan en una relación típica de derecho» (KOJÈVE, 1981: 19) ${ }^{6}$. La tipología que KOJÈvE expone se inspira en PlaTÓN (ideas), WEBER (tipos ideales) y HuSSERL (descripción eidética) ${ }^{7}$, si bien difiere de sus tres antecesores por mantener una perspectiva histórica según la cual la comprensión y realización del derecho evolucionaría hasta llegar al punto, término final de la evolución, en que su esencia coincidiría con su existencia. Obviamente en el trasfondo de esta perspectiva está HEGEL —aunque interpretado y reelaborado por KOJÈvE (JARCZYK y LABARRIÈRE, 1996)—. Pero, asimismo, la descripción fenomenológica ha de permitir una crítica de los usos inadecuados del término «derecho». El objeto de tal descripción es dar con un criterio que sirva para discriminar caso por caso si existe o no propiamente una relación jurídica. Ahora bien, el Esquisse d’une phénoménologie du droit no puede ser más que un boceto. No nos da ninguna explicación ontológica o metafísica del derecho - sino solo fenomenológica - por tratarse de una reflexión todavía imperfecta, y sobre todo porque el Estado universal y homogéneo, único criterio - no transhistórico, sino inmanente a la historia— que permitiría juzgar (hegeliana-

5 A. KoJève, Outline of a Phenomenology of Right; translated, with and introductory essay and notes, by B.-P. Frost and R. Howse, edited by B.-P. Frost, Lanham: Rowman \& Littlefield Publishers [2000] 2007.

6 «Pour arriver à une telle définition "béhavioriste" du phénomène juridique, voyons comment agissent les bommes lorsqu'ils se trouvent dans un rapport typique de droit».

7 «Il s'agit de trouver l' "Idée" (PLATON), l'Idealtypus" (Max WEBER), le "Phénomène” (HuSSERL), etc., de l'entité étudiée, en analysant un cas concret particulièrement net, typique, spécifique, pur. Il faut découvrir en d'autres termes le contenu qui fait que le cas donné est un cas de droit par exemple, et non de religion ou d'art, etc. Et l'ayant découvert, c'est-à-dire ayant trouvé l' "essence" (WESEN) du phénomène, il faut le décrire d'une façon correcte et complète, cette description de l'essence n'étant rien d'autre que la définition du phénomène en question» (KOJÈVE, 1981: 10). 
mente) el desarrollo del derecho aún no se habría realizado completamente (KoJÈve, 1981: 11, 13 y 17).

Ante todo, KoJÈve subraya el logro fundamental del derecho europeo moderno: «Porque el derecho europeo moderno tiene como principio que el ser humano (a saber, el homo sapiens) por el mero hecho de su existencia es un sujeto de derecho» (KoJÈvE, 1981: 41-2) ${ }^{8}$. Este principio reviste una enorme importancia, y no es nada difícil sacarlo a la luz de los fundamentos de la filosofía del derecho del que es su máximo referente (HEGEL, 1993). Paralelamente a dicha realización del derecho, el proceso histórico antropogénico da como resultado la figura del «ciudadano» del Estado universal y homogéneo (la ciudadanía cosmopolita), que desde la Introduction à la lecture de Hegel ve KOJĖvE como ya acontecido, al menos formal y potencialmente. En su argumentación, la universalización del derecho es una pieza clave de esa evolución histórica. Por otra parte, los derechos carecen de sentido fuera del mundo humano: animales o cosas solo son objeto de derecho en la medida de sus relaciones con los seres humanos, esto es, en la medida en que se encuentran bajo su cuidado y protección (KoJÈVE, 1981:34). Todo ello se apoya en una antropología filosófica de raíz hegeliana: el derecho avanza en paralelo con la historia de la emancipación, a saber, la historia del esclavo que, según HEGEL, se emancipa del amo para llegar a ser ciudadano.

Tres son las formas que adopta la relación jurídica9 : a) tener el derecho; $b$ ) no tener el derecho, y $c$ ) tener el deber de hacer u omitir determinada acción. Como $b$ y $c$ en realidad son equivalentes (puesto que, por un lado, no tener el derecho a hacer $x$ equivale a tener el deber de no hacer $x$; $y$ por el otro, no tener el derecho de no hacer $x$ equivale a tener el deber de hacer $x$ ) y tener el deber de hacer algo implica por necesidad tener también el derecho (si bien no se identifican), en realidad solo $a$ es pertinente. $Y$ es que donde haya alguien que tenga el derecho de hacer u omitir $x$, habrá derecho. Lo aclara perfectamente una serie de ejemplos (o casos prototípicos a la manera de paradigmas) que muestran la relación jurídica como resultado de la interacción entre tres elementos [como reza el adagio romano, tres faciunt collegium (KoJÈvE, 1981: 75)]: por un lado, dos agentes, A y B, que interactúan, y por el otro lado, $\mathrm{C}$, que dirime si existe o no el derecho a determinada acción a raíz de su intervención en el conflicto entre A y B. Antes de adentrarnos en los detalles de la formación del derecho es importante destacar que, desde un punto de vista metodológico, la descripción kojeviana del derecho no es ni una definición a priori del derecho ni una recolección a posteriori de todas las instancias denominadas «derecho».

Sería fácil dar una definición arbitraria del Derecho, aun con el riesgo de negarse a llamar «jurídico» a todo cuanto, por lo demás, así se denomina pero no se ajusta a la definición elegida. Solo que tal definición tendrá poco interés, pues es imposible hacer remilgos a las enseñanzas contenidas en el lenguaje y la historia. Si algo es —o fue— llamado «derecho», lo más probable es que no haya sido por azar. Pero, por otra parte, es materialmente imposible el reunir en una sola definición todo lo que se ha llamado «derecho» en todo momento y lugar: este contenido sería disparatado (KoJĖve, 1981: 9-10) ${ }^{10}$.

\footnotetext{
8 «[C]ar le droit européen moderne a pour principe que par le fait seul de son existence, l'être bumain (s'entend l'Homo sapiens) est un sujet de droit».

9 La explicación detallada se encuentra en las $\$ \$ 5-19$ del Esquisse d'une phénoménologie du droit.

10 «Il serait facile de donner du Droit une défnition arbitraire, quitte à refuser d'appeler "juridique" tout ce qui est appelé ainsi par ailleurs, mais ne cadre pas avec la définition choisie. Mais une telle définition aurait peu d'intérêt, car il est impossible de faire simplement fi des enseignements impliqués dans le langage et dans l'bistoire.
} 
Cabe una «vía intermedia» entre ambos métodos, que KoJÈvE se propone mediante el uso de paradigmas para definir y describir el derecho en cuanto derecho, autónomo y distinto de otras instancias tales como la religión, la moral o la política. Esta «vía intermedia» pondría de manifiesto la «autonomía del derecho», de modo semejante a cómo Carl ScHMiTT en El concepto de lo político había sostenido la autonomía de la política. En segundo lugar, KoJÈvE repasa el nacimiento del derecho a partir de la hegeliana lucha por el reconocimiento. Al mismo tiempo estudia su evolución histórica con relación a dos formaciones históricas de la justicia: la justicia de la igualdad (aristocrática) y la justicia de la equivalencia (burguesa), cuya síntesis sería la justicia de la equidad del ciudadano (propia del Estado universal y homogéneo). En tercer y último lugar, brinda una clasificación de los fenómenos jurídicos y una descripción, poco ortodoxa pero de gran interés filosófico, del derecho internacional, el derecho privado y el derecho público. Su objetivo es describir la esencia del derecho en su realización última (en el Imperio socialista o Estado universal y homogéneo) que satisfaría el deseo humano de reconocimiento, con lo cual se daría por finalizada la evolución histórica de la humanidad ${ }^{11}$.

\section{LA NOCIÓN KOJEVIANA DE DERECHO}

Por supuesto se debe a su obra anterior, Introduction à la lecture de Hegel cuyo hilo conductor es la dialéctica del amo y el esclavo, la importante función que esta última desempeña en la formación e historia del derecho desarrollada en la segunda parte del Esquisse. Ahora bien, ante todo es preciso atender a un elemento esencial de la kojeviana filosofía del derecho, cuyo mero planteo ya entraña por sí solo una original heterogeneidad con respecto a las posiciones más extendidas en dicha filosofía. Como ha defendido François TERRÉ (2011: 422), KoJÈVE aporta una luz singular que, siendo algo periférica, se demuestra de la mayor utilidad para reflexionar filosóficamente sobre el derecho. En efecto, resulta muy importante la intuición de que, para que se dé propiamente una «situación jurídica» o «relación de derecho», hace falta la presencia de una terceridad o tercero. La relación de derecho implica, pues, tres miembros: A y $\mathrm{B}$ son dos agentes que interactúan, lo que motiva - pero no determina- la intervención de un tercero, $\mathrm{C}$.

En resumen, el tercero $C$ no interviene sino en la medida en que la conducta (acción) de B (voluntad, acto o fin) tienda a suprimir la conducta (acción) de B (voluntad, acto o fin), esto es, en la medida en que la acción de B sea una verdadera «reacción», esto es, en la medida en que haya una «interacción» entre A y B (KoJève, 1981: 67) ${ }^{12}$.

Una acción se compone de tres elementos: 1) la «voluntad» (resolución interna o declaración expresa de hacer o no hacer algo); 2) el «acto» en sí u objeto de la volun-

Si quelque chose est —ou a été- appelé "Droit", il est plus probable que ceci n'a pas été fait par hasard. Mais d'autre part il est matériellement impossible de réunir en une seule définition tout ce qui est appelé "Droit" à un moment quelconque et quelque part: ce contenu serait trop disparate».

${ }_{11}$ Esta es una de las tesis rectoras de la Introduction à la lecture de Hegel (1968) de KojÈvE.

12 «En bref, le tiers $C$ n'intervient que dans la mesure où le comportement (l'action) de B (volonté, acte ou but) tend à supprimer le comportement (l'action) de A (volonté, acte ou but), c'est-à-dire dans la mesure où l'action de B est une véritable "réaction", c'est-à-dire dans la mesure où il y a une "interaction" entre A et B». 
tad, y 3) la meta o finalidad del acto en cuestión (FrOsT, 1999: 603). Estos tres elementos pueden darse por separado. Si, por el contrario, se dan conjuntamente se podrá hablar con propiedad de una «acción voluntaria», que es justo la materia sobre la que opera el derecho. KoJÈvE sostiene que la situación jurídica se revela cuando, a raíz de la interacción entre $\mathrm{A}$ y $\mathrm{B}$, interviene $\mathrm{C}$ para impedir la reacción de $\mathrm{B}$ a la acción de $\mathrm{A}$ (KoJÈVE, 1981: 28); y, por tanto, garantiza el derecho de A de hacer o de no hacer algo. Es en este punto, en $\$ 7$ (KoJÈVE, 1981: 22 y ss.), donde KoJĖVE describe las situaciones paradigmáticas que hacen comprensible a partir de ejemplos típicos la relación jurídica. El primero de tales ejemplos tiene que ver con el dinero. Supongamos que A quiere recibir una determinada cantidad de dinero de B. Esta acción de A tiene como consecuencia una reacción de B. Pueden abrirse entonces dos escenarios: 1) que B impida a A obtener esta suma de dinero, o 2) que A, que es más fuerte, obtenga el dinero de B por coacción. En ninguno de ambos casos, por lo dicho, se tratará propiamente de una relación de derecho. Tampoco lo será si al cabo se produce un robo o una donación voluntaria. Nada de todo ello pone de manifiesto el derecho como tal. Pero la situación cambiará radicalmente si aparece un $\mathrm{C}$ para garantizar a $\mathrm{A}$ la obtención de la suma. Ahora bien, la aparición de $\mathrm{C}$ deberá tener la siguiente forma:

A actúa con el fin de obtener el dinero; B reacciona para no soltarlo; pero una tercera persona $\mathrm{C}$ interviene y anula la reacción de $\mathrm{B}$ de modo que $\mathrm{A}$ recibe el dinero sin haber tenido que hacer esfuerzo alguno para obtenerlo, ya que la reacción de $\mathrm{B}$ que $\mathrm{A}$ hubiera debido anular ha sido anulada por la intervención de C. Hay que añadir que A y B son indiferentes, esto es, unos desconocidos para $\mathrm{C}$, y que $\mathrm{C}$ no está personalmente interesado en que el dinero quede en manos de $\mathrm{B}$ o pase a $\mathrm{A}$. En este caso puede decirse con certeza que asistía a A el derecho de tomarlo. Cabe suponer, por ejemplo, que A quería recuperar el dinero que había dejado en poder de $\mathrm{B}$ y que $\mathrm{C}$ es el agente judicial que ha efectuado la operación por cuenta de A al no querer B llevarla a cabo espontáneamente (KoJĖve, 1981: 22-23) ${ }^{13}$.

KoJÈvE añade cuatro ejemplos más: A y B están sentados en el banco de un parque de manera que $B$ proyecta su sombra sobre $A$, y $A$ le ruega que se aparte; $B$ es un vecino de $A$ que hace ruido por las noches y $A$ actúa para que $B$ cese de hacerlo; $B$, madre de un niño que se ha caído en el río y está ahogándose, pide ayuda a $A$, que sabe nadar, para salvarlo; y por último, $\mathrm{A}$ ataca a $\mathrm{B}$ y $\mathrm{B}$ reacciona para detener los golpes. «Es inútil alargar esta lista de ejemplos» (KoJĖVE, 1981: 23). En todos ellos aparece la figura de $\mathrm{C}$ como garante de que $\mathrm{A}$ tiene o no tiene el derecho de hacer o no hacer determinada acción. Lo más relevante, empero, es que $\mathrm{C}$ actúa desinteresadamente. Así que $\mathrm{C}$ tenga de antemano una preferencia por A o por B podrá afirmarse que no se da el fenómeno del «derecho». Para que el fenómeno jurídico aparezca resulta absolutamente necesario que la intervención de $\mathrm{C}$ sea «imparcial» y «desinteresada». Imparcial: $\mathrm{C}$ no siente ninguna preferencia ni por A ni por B; luego su intervención tendría exactamente las mismas características incluso si A y B se intercambiaran los papeles, de manera que A fuese B y B fuese A. Desinteresado: C modifica objetivamente el estado de A y B, pero

13 «A agit en vue d'obtenir l'argent; B réagit de façon à le garder; mais une troisième personne $C$ intervient, annule la réaction de $B$ de sorte que $A$ reçoit l'argent sans avoir eu à faire d'effort pour l'avoir, la réaction de $B$ que $A$ aurait dî annuler ayant été annulée par l'intervention de $C$; ajoutons que $A$ et $B$ sont indifférents, voir inconnus à $C$ et que $C$ n'est pas intéressé personnellement à ce que l'argent reste chez $B$ ou passe à $A$. Dans ce cas on peut dire avec certitude que $A$ avait droit de le lui prendre. On peut supposer par exemple que $A$ voulait reprendre à $B$ l'argent qu'il avait déposé chez lui et que C était l'buissier qui a fait l'opération pour le compte de $A, B$ ne voulant pas s'exécuter spontanément». 
no modifica el suyo propio (KoJÈvE, 1981: 77). Puesto que la idea de «desinterés» no queda lo bastante explicada con ese rasgo, KOJÈvE añade que $\mathrm{C}$ es desinteresado en tanto que $\mathrm{C}$ podría ser cualquiera y aun así no se modificaría su tipo de intervención; es decir, que cualquier otro intervendría de forma idéntica a la de $C$. En suma, la razón de ello es que la intervención de $\mathrm{C}$ no tiene otro objeto o interés que la Justicia. De ahí que se la califique de «desinteresada» y, por extensión, también de «imparcial».

Además la intervención de $\mathrm{C}$ debe ser «consciente y libre». La conciencia se revela fenomenológicamente en el hecho de que, sin la interacción de A y B, la intervención de $\mathrm{C}$ no hubiera tenido lugar. La libertad se revela en el hecho de que la intervención de $\mathrm{C}$ no se deriva mecánicamente de la interacción de $\mathrm{A}$ y $\mathrm{B}$, ya que tal intervención podría no haberse dado (es contingente). La intervención de $\mathrm{C}$ está condicionada por la interacción de A y B, pero no está determinada por ella. Esto es fundamental porque el criterio de intervención del tercero no se ciñe a los simples elementos que se ponen en juego en la interacción entre $\mathrm{A}$ y $\mathrm{B}$, sino que $\mathrm{C}$ tiene, por decirlo así, sus propias razones para intervenir entre A y $\mathrm{B}$. Pues bien, es la intervención y no la interacción per se lo que pone de manifiesto el fenómeno jurídico (KoJÈVE, 1981: 69-73). Aplíquese al contexto político (el Estado) en que aparece la relación de derecho:

Para que exista una situación jurídica hace falta (1) que el Estado se relacione con amigos políticos, que A y B, que interaccionan, sean ciudadanos, y (2) que A y B estén en interacción no política entre sí, de modo que el Estado pueda desinteresarse de la naturaleza de su interacción y no ser parte al dejar de ser juez (KoJÈve, 1981: 147) ${ }^{14}$.

Todo ello solo es posible si se comprende que $\mathrm{C}$ tiene su propio criterio a la hora de intervenir. Este criterio es lo que KoJĖve llama «la idea o ideal de Justicia». Por tanto, la motivación de $\mathrm{C}$ se basa en algo que podríamos llamar un «sentido de la justicia» ${ }^{15} \mathrm{y}$ un deseo de realización de un determinado ideal de justicia. El contenido de esta idea o ideal de justicia no es transhistórico, sino que está siempre ubicado históricamente. La fenomenología del derecho, por tanto, está también incardinada y contextualizada históricamente. KoJÈvE no duda en aclarar que la idea o ideal de justicia varía con el tiempo y que depende de la sociedad y del grupo que en ella ejerce el liderazgo: es su idea de justicia la que rige. Así pues, en realidad, el tercero $\mathrm{C}$ a lo largo de la historia no pudo ser cualquiera, o mejor dicho, solo un cualquiera de una época determinada y de un grupo exclusivo concreto (la élite dirigente) en una sociedad particular. No sería hasta el advenimiento de un Estado universal y homogéneo que, de hecho, $\mathrm{C}$ podría ser realmente cualquiera. Solo en estas circunstancias la existencia y la esencia del derecho se recubrirían totalmente.

La expresión «Estado universal y homogéneo» puede parecer algo extraña y contraintuitiva, pero de hecho capta a la perfección dos rasgos fundamentales de la rea-

14 «Pour qu'il y ait une situation juridique, il faut donc (1) que l'État se rapporte à des amis politiques, que $A$ et $B$, qui sont en interaction, soient des citoyens, et (2) que $A$ et $B$ soient en interaction non politique entre eux, de sorte que l'État puisse se désintéresser de la nature de leur interaction et ne pas être partie là où il est cessé d'être Juge».

${ }_{15}$ Para una versión normativa y no histórica de la idea o sentido de la justicia, vid. J. RAWLS, $A$ Theory of Justice, Oxford: Oxford University Press, especialmente cap. 8, sec. 69-77, 1980. Aunque se trata de dos contextos diferentes, tanto la «idea o ideal» de la justicia kojeviana como el «sentido» de la justicia rawlsiano dan contenido a principios formales. La fundamentación de la «idea o ideal» de la justicia es en KojÈve muy deudora de la filosofía de la historia hegeliana, con lo cual obviamente dista mucho de la posición de RAWLS. 
lización de la idea o ideal de la justicia. Primero, la universalidad: si una sociedad es realmente universal y engloba a la humanidad entera, se puede eliminar de la definición de derecho la circunstancia de que $\mathrm{C}$ forma parte de una sociedad particular. Solo entonces el desinterés de $\mathrm{C}$ será auténtico, porque no se basará en su pertenencia a una sociedad concreta. Segundo, la homogeneidad: este rasgo del estado final es de una relevancia capital. Significa que no hay un «grupo exclusivo» que detente el poder en una sociedad determinada, esto es, no hay un cúmulo de intereses particulares que obren en contra del resto de la sociedad, a saber, de aquellos que no forman parte del «grupo exclusivo» siendo miembros del «grupo excluido». Por tanto, «homogeneidad» no tiene el sentido de negación de toda diversidad. El propio KoJÈvE indica que, sin duda, en el Estado universal y homogéneo persistirá un montón de diferencias individuales insuperables. «[P]ues, de hecho, la homogeneidad nunca es absoluta: hay diferencias de edad, sexo, carácter, etc., y por consiguiente agrupaciones subestatales» (KoJÈVE, 1981: 152 ${ }^{16}$. Universalidad y homogeneidad combinadas garantizan la ausencia de enemigos exteriores (universalidad) y enemigos interiores (homogeneidad), con lo que el estado final logra una estabilidad suficiente como para que se dé por supuesta su continuidad e inmutabilidad en el tiempo. Justo en este momento podrá ser $\mathrm{C}$ cualquiera, imparcial y desinteresado.

Aunque Kojève no reflexione lo suficiente sobre las implicaciones de esta afirmación, dichas implicaciones son extraordinarias. Si solamente en el final de la historia $\mathrm{C}$ podría ser realmente cualquiera, entonces todos y cada uno de los individuos estarán de acuerdo con los principios jurídicos que determinan qué opciones específicas se consideran criminales, y el estado final y solo el estado final dará expresión a un sistema de derecho universalmente aceptado y plenamente satisfactorio (FrOST, 1999: 608) ${ }^{17}$.

Todas las concepciones del derecho anteriores en la historia serían todavía incompletas, no habrían conseguido realizar la esencia del derecho. Y ello debido a que serían relativas a los intereses de las élites gobernantes en una sociedad y estarían en función de una época determinada. A diferencia de las concepciones más formalistas del derecho, KoJÈVE apunta a la necesidad de considerar el derecho como un producto histórico, relativo a las sociedades formadas en momentos históricos definidos y regidas además por intereses de los grupos gobernantes. El ideal de justicia, pues, no es neutro en la historia, sino que se ha visto siempre teñido por su entorno. El único modo de romper con el horizonte histórico consiste precisamente en culminar la evolución histórica, siendo su culminación el estado final o universal y homogéneo. El Esquisse es deudor de la Introduction en este punto crucial.

Por último, debe advertirse que la relación jurídica, al implicar por fuerza una terceridad, elimina de antemano como situaciones de derecho todas las intervenciones en que el Estado es juez y parte al mismo tiempo, esto es, el derecho público como tal. «En cuanto al "Derecho" público, no es un Derecho ni en tiempos de guerra ni

\footnotetext{
${ }^{16}$ «[C]ar en fait l'homogénéité n'est jamais absolue: il y a différences d'âge, de sexe, de caractère, etc., et par conséquent des groupements cis-étatiques».

17 «Although Kojève only briefly elaborates upon the implication of this claim, these implications are nothing less than extraordinary. If $C$ could be anyone at all only at the end of history, then each and every individual would agree with the juridical principles which determined what specific options were considered criminal, and the end state and the end state alone would give expression to a universally accepted and fully satisfying system of right».
} 
en tiempos de paz» (KOJÈVE, 1981: 152, nota al pie). François TERRÉ se ha demorado en este punto, que llama mucho la atención ciertamente. El argumento kojeviano no deja lugar a dudas: solo hay derecho cuando un $\mathrm{C}$ imparcial y desinteresado interviene entre A y B. Si el Estado como tal es juez y parte afectada al mismo tiempo, entonces la relación es política, pero no jurídica. Afirma TERRÉ:

Así, pues, en el análisis que efectúa KoJÈve «allí donde se trata de relaciones de interacción con el propio Estado, no hay elemento jurídico en absoluto». Con más razón resulta ser así cuando se pasa del Derecho administrativo al Derecho constitucional: «En la medida en que la Constitución fija el estatus del Estado y de los ciudadanos, esta no tiene nada de jurídica. Es una ley puramente política del Estado, es decir, una ley que el gobierno crea como quiere y que este puede cambiar como quiera [...]. Si el ciudadano actúa como ciudadano entonces, por definición, este no puede entrar en conflicto con el Estado». Pese a la concurrencia de tantas fórmulas — a simple vista paradójicas, excesivas y radicales- el jurista de nuestra época debe especialmente interrogarse, no obstante, acerca de los excesos del todo jurídico, sobre todo ante el espectáculo de zonas intermedias respecto a las que el modo de operar del filósofo resultaría ser particularmente esclarecedor y operacional (TERRÉ, 2011: 421-22).

El tipo ideal kojeviano de relación de derecho o situación jurídica no encaja en el derecho administrativo y aun menos en el derecho constitucional. ¿Significa esto que deben abandonarse tales denominaciones? Es decir, ¿significa esto que el derecho administrativo y el constitucional no son propiamente «derecho»? No exactamente. Lo interesante del análisis de KOJÈVE consiste, precisamente, en cuestionar esas zonas intermedias del derecho en que la imparcialidad y el desinterés, criterios ambos de lo jurídico, pueden estar bajo sospecha por cuanto la relación que el Estado establece con el ciudadano no se basa en un tercero imparcial, sino en la unilateralidad de uno de los dos agentes, en este caso el Estado como juez y parte al mismo tiempo. En definitiva, lo interesante de KoJÈvE es que nunca cruza el umbral, que hace visible, de la relación entre derecho y política. Siempre tiene presente el elemento político en el derecho, pero sin reducir este a la política.

\section{EL SUPUESTO DERECHO INTERNACIONAL}

Una de las distinciones que KoJÈvE aplica al derecho es la de «derecho en potencia» y «derecho en acto», de evidente cuño aristotélico. La potencialidad del derecho implica que este se halla en proceso de devenir mientras que la actualidad del derecho implica, en cambio, que ya ha devenido. Semejante distinción resulta fundamental para comprender la relación jurídica entre Estados; tiene, pues, consecuencias en el ámbito del derecho internacional.

La diferencia entre potencialidad y actualidad se da en función de lo irresistible que sea la intervención de $\mathrm{C}$ en el conflicto entre $\mathrm{A}$ y $\mathrm{B}$. Si la intervención de $\mathrm{C}$ es realmente irresistible, puede anular la reacción de $\mathrm{B}$ contra el derecho de $\mathrm{A}$ de hacer u omitir $x$. En la medida en que la intervención de $\mathrm{C}$ no sea irresistible y $\mathrm{B}$ tenga la posibilidad de reaccionar contra A, se dirá que el derecho solo existe en potencia. Por el contrario, si la acción de $\mathrm{C}$ es irresistible, entonces el derecho se habrá actualizado. Conviene remarcar que dicho criterio es estrictamente formal: no da cuenta de los 
contenidos y objetos del derecho, sino de la forma en que una terceridad efectiva puede intervenir de manera irresistible (sin tropezar con una resistencia insuperable) en la interacción de A y B. Los contenidos del derecho dependen de la idea o ideal de Justicia; se trata de un contenido histórico que responde a la interpretación kojeviana de la hegeliana dialéctica del amo y el esclavo a lo largo de la historia. Nos detendremos más adelante sobre ello. Por de pronto lo decisivo es que el derecho internacional siempre se halla en potencia a menos que exista un organismo internacional que pueda actuar como tercero o C de forma irresistible; léase un Estado mundial, que KOJÈVE vincula al Estado universal y homogéneo. La universalización del derecho implica, por tanto, la desaparición de las soberanías estatales y, justamente por ello, cuestiona el ser mismo de lo político entendido como potencial relación de enemistad en un cuadro de inspiración hobbesiana y schmittiana. KoJève ilumina el derecho con una nueva luz al ponerlo en relación con su potencial de despolitización. En donde exista una relación jurídica se desvanecerá la relación propiamente política, que está basada en los opuestos amigo y enemigo. «Pero doy por supuestas estas dos categorías fundamentales, específicamente políticas» (KoJÈVE, 1981: 144 y en la nota al pie cita «Karl Schmidt» [sic], Der Begriff des Politischen). La influencia de Carl ScHMITT en el planteamiento kojeviano es manifiesta.

Se trata de entender que el Estado es una asociación política autónoma. En lo sucesivo, al existir una pluralidad de estados existe también una pluralidad de derechos. Pero el derecho está solo en potencia en tanto que cualquiera puede huir a un Estado donde la ley no le alcance; por ende, esta ley no es «irresistible». Con la kojeviana definición del derecho en mano es fácil reparar en que hasta los tratados de extradición y otras fórmulas de cooperación interestatal, en la medida en que dependen de la voluntad política de los Estados firmantes, seguirán siendo «derecho en potencia». Luego su relación no será jurídica sino política, sin menoscabo de que pueda suponer -y supone de hecho- algunos avances en el proceso de juridificación mundial. Ahora bien, tal proceso solo podrá darse por culminado con el advenimiento del Estado universal y homogéneo. Muchos y plausibles son los argumentos contra la existencia de un estado mundial (en terminología kojeviana, un «estado final»). Entre los mismos destaca algo que ya KANT había señalado: el peligro de una tiranía mundial sin contrapeso u oposición política algunos. El estado mundial pudiera traducirse en una efectiva liquidación de la ciudadanía, entendida esta según el modelo liberal y republicano.

Ahora bien, si el Estado es realmente universal y bomogéneo, entonces la ciudadanía será realmente universal y homogénea. No se trata de una simple tautología. Antes bien, logra evitarse el potencial bélico de los conflictos entre Estados, siempre dentro del esquema schmittiano amigo-enemigo, con lo cual la guerra pasa a ser imposible al universalizarse el Estado. La homogeneidad no debe entenderse como uniformización social, sino como supresión de la diferencia entre el grupo exclusivo de gobernantes y el grupo excluido de gobernados (esto es, como supresión de la diferencia entre clases). Entonces, lo reiteramos, cualquiera podrá ejercer de C desinteresado e imparcial que juzgue sobre la interacción de distintos agentes. Tampoco significa ello que todo C juzgará del mismo modo; así como las peculiaridades individuales de los ciudadanos del Estado universal y homogéneo persistirán (de ahí la insistencia de KOJÈvE en el fe- 
nómeno del esnobismo en varios pasajes de su obra) ${ }^{18}$, persistirá también la aplicación prudencial, contextual y en cada caso particular de C. Los dictámenes de C, por tanto, no serían idénticos para cada caso, pero sí las reglas de justicia en que se inspiraría. En suma, no habrá en el Estado universal y homogéneo distintas reglas de justicia en función de cada Estado, sino que las reglas de la justicia se habrán universalizado y actualizado. Si en la primera definición de derecho $\mathrm{C}$ puede ser cualquiera dentro de un grupo exclusivo en una sociedad dada en un momento determinado, según la definición final de derecho ocurre todo lo contrario:

Si una sociedad es homogénea, podremos suprimir las palabras «de un grupo exclusivo». Si es universal, podremos suprimir las palabras «de una sociedad dada». Si es homogénea y universal, podremos eliminar todo el paréntesis, es decir, todas las restricciones introducidas en este párrafo. Entonces la definición de la esencia del derecho se aplicará también a la existencia del derecho, precisamente porque existencia y esencia serán una: la esencia de la ley estará plenamente realizada y la existencia estará enteramente penetrada por la plenitud de la esencia jurídica. La justicia se realizará plenamente en y por el derecho, porque la Justicia determinará a toda existencia humana (KoJÈvE, 1981: 94) ${ }^{19}$.

En puridad, no cabe admitir fácilmente que el Estado universal y homogéneo sea de veras un Estado. Más bien parece tratarse de una sociedad organizada mediante un aparato jurídico. Si por «Estado» entendemos una administración (evidentemente la administración de la justicia inclusive), entonces el Estado universal y homogéneo es de hecho un «Estado» — en la medida en que todos los ciudadanos serán «administrados» por él一. Lo será en términos weberianos, por así decir. No obstante, al desaparecer lo político por saturación, en otro sentido el Estado (a partir de su significado histórico) está condenado a desaparecer. El Estado universal y homogéneo rompe con la existencia de Estados como expresión de lo político porque precisamente acaba con lo político concebido en el marco de la relación «amigo-enemigo». Al no haber una pluralidad de Estados (el «pluriverso» de Sснмітт) tampoco hay la posibilidad de enemistarse, de forma que se llega a una situación de «universalidad» despolitizada. Y no cabe hablar de «neutralidad» ya que ni siquiera existiría la posibilidad de un conflicto; más bien se trata de neutralización. Lo interesante en KoJÈvE es que la despolitización corre pareja a la juridificación: la autonomía y especificidad (es decir, la irreductibilidad a otros ámbitos) de lo jurídico se muestra análoga a la autonomía y especificidad de lo político en SCHMITT. De hecho, KOJÈvE la concibe como alternativa a la resolución de los conflictos humanos que, en SСНMiтT, lleva en casos extremos a la guerra para medir las diferencias ${ }^{20}$. Ahora, por el contrario, es el arbitraje del tercero lo que resuelve los conflictos entre miembros de la especie bomo sapiens sin tener que

18 Sobre todo en la famosa nota al pie añadida en la segunda edición de la Introduction à la lecture de Hegel (KoJÈve, 1968: 436-437).

19 «Si une Société est homogène, on pourra supprimer les mots: "d'un group exclusif". Si elle est universelle, on pourra supprimer les mots "d'une Société donnée". Si elle est homogène et universelle, on pourra supprimer toute la parenthèse, c'est-à-dire toute la restriction introduite dans ce paragraphe. La définition de l'essence du droit s'appliquera alors aussi à l'existence du Droit, précisément parce que l'existence et l'essence ne feront plus qu'un: l'essence du droit sera pleinement réalisée et l'existence sera entièrement pénétrée par la plénitude de l'essence juridique. La justice sera pleinement réalisée dans et par le Droit parce que toute l'existence humaine sera déterminée par la Justice».

${ }_{20}$ Para una contraposición y debate entre los planteamientos de Kojève y SCHMiTT, vid. A. Kojève, 2001: «Alexandre Kojève-Carl Schmitt Correspondence and Alexandre Kojève, Colonialism from a European Perspective», Interpretation, vol. 29, n. 1, 91-130. 
recurrir a las armas. La juridificación, por tanto, es algo así como una pacificación. Sin duda parten ambos autores de una tendencia al conflicto de orden antropológico, pero KoJĖVE la supera con un giro hegeliano desde el potencial irénico del derecho ${ }^{21}$.

KoJÈVE brinda una concepción del derecho internacional público como potencialidad pacificadora que superaría la beligerancia entre Estados. Pero el derecho internacional público no es más que un derecho facultativo (facultatif), esto es, en lenguaje de nuestro autor, un derecho en potencia. $\mathrm{O}$ en sus propias palabras:

Cuando dos Estados se someten al arbitraje de un tercero (o - lo que es lo mismo- al arbitraje de un tribunal internacional, de una SDN [Sociedad de las Naciones], o incluso de la «opinión mundial»), se da una situación jurídica real, ya que todos sus elementos (el juez y las partes) son reales. Pero desde el momento en que el arbitraje es facultativo, este derecho real solo existe en potencia. Los dos Estados-parte y el Estado-árbitro forman así una «Sociedad» que implica un derecho real. Sin embargo, cada miembro puede abandonar esta Sociedad y formar una sociedad «afuera». El derecho real de esta Sociedad no se actualiza pues necesariamente. Es un derecho real en potencia. Y es por eso que el derecho internacional contemporáneo debe modificarse para llegar a ser real y en acto (KOJÈVE, 1981: $131-132)^{22}$.

Esta modificación del derecho internacional público que plantea KoJÈVE no es otra cosa que la realización efectiva de una Sociedad mundial que no permita el abandono. $\mathrm{O}$ dicho de otro modo: se alcanza una situación en que las resoluciones de $\mathrm{C}$ (como árbitro entre A y B) son realmente irresistibles. Una de las fórmulas que KoJÈVE sugiere en su concepción de la expansión jurídica es su paso por una «federación» de Estados y un cosmopolitismo cívico (MURPHY, 2011). Su federalismo es pues un medio para alcanzar la universalidad y homogeneidad en la administración de la justicia:

Como entidad política, el Estado tiende a expandirse por conquista; lisa y llanamente, trata de absorber a los estados extranjeros. Pero como entidad jurídica, el Estado se conforma con imponer en el extranjero su derecho interno. En otras palabras, tiende a crear una Federación de Estados o un Estado federal, transformándose él mismo en uno de los Estados federados. Tal Federación tiene como base y resultado la existencia de un derecho único, común a todos los Estados federados, e implica —en su aspecto de «Derecho público»— un elemento de «derecho federal» que regula las relaciones de los Estados Federados entre sí; en particular, la organización federal de la justicia. Si la Federación no es universal, y si tiene Estados-enemigos en el exterior, deberá organizarse como Estado propiamente dicho (federal). Sus elementos integrantes - los Estados federados - también tendrán enemigos; por consiguiente, serán Estados. Pero tendrán siempre enemigos comunes y deberán reconciliarse con ellos en común: no serán, por tanto, Estados soberanos, sino Estados federados. Solo la Federación tenderá a expandirse tanto como le sea posible. En última

21 «En fait un État national ne réussit jamais ni à absorber tous ces ennemis, ni à s'isoler complètement d'eux. La limite n'est atteinte que par l'État universel ou l'Empire. Mais alors il n'y a plus d' "ennemis", c'est-à-dire plus de domaine politique. L'Empire, en actualisant complètement la relation politique fondamentale d'amis-ennemis, épuise la "puissance" politique. Or l'acte s'annule au moment où il épuise sa puissance. L'Empire n'est donc plus une entité politique au sens propre du mot : il n'a pas d'bistoire politique» (KOJÈve, 1981: 147).

${ }_{22}$ «Quand deux États se soumettent à l'arbitrage d'un troisième (ou —ce qui est la même chose-à l'arbitrage d'un Tribunal international, d'une S.D.N. [Société des Nations], ou même de l'"opinion mondiale”), il y a une situation juridique réelle, puisque tous les éléments (le Juge et les Parties) sont réels. Mais, du moment que cet arbitrage est facultatif, ce Droit réel n'existe qu'en puissance. Les deux États-parties et l'État-arbitre forment bien une "Sociétê" impliquant un droit réel. Mais chaque membre peut quitter cette Société et former une Société "en debors". Le Droit réel de cette Société ne s'actualise donc pas nécessairement. C'est un Droit réel en puissance. Et c'est pourquoi le Droit international contemporain doit se modifier pour devenir réel et en acte». 
instancia, abarcará toda la humanidad. Entonces dejará de ser un Estado en el sentido propio de la palabra al no tener enemigo exterior. Y los Estados Federados también dejarán de ser verdaderos Estados. La Federación se convertirá entonces en una simple unión jurídica mundial (al menos en su aspecto jurídico, que no es el único) (KOJÈVE, 1981:391) ${ }^{23}$.

Lo interesante y verdaderamente actual de la filosofía del derecho de KoJÈVE es que el Estado universal se convierte en una exigencia normativa para lograr el irénico objetivo de la paz entre naciones. La universalidad de tal sociedad imposibilitaría de facto su abandono. En cuanto a la homogeneidad, la exigencia de la misma es resultado de la base sociológica de raíz hegelomarxista en que se apoya nuestro autor. Su punto de partida es la constatación de que la posibilidad de conflictos no solo se da en un pluriverso de sociedades nacionales que denota su estado de naturaleza, sino que en el propio interior de las sociedades se produce necesariamente una separación entre grupos antagónicos en la forma de lo que KoJĖve llama «grupo exclusivo» y «grupo excluido». Aun concediendo la existencia de posibles fricciones en el seno de los grupos antagónicos, de ordinario el grupo excluido opondrá resistencia al poder - $\mathrm{o}$ incluso cabe desencadenar una revolución contra el orden establecido por y para el grupo exclusivo- - La homogeneidad social, en cambio, eliminaría la formación de estos dos grupos antagónicos, con lo que se alcanzaría entonces la paz social en el interior de la sociedad en cuestión. El Estado universal y homogéneo, por tanto, es la superación efectiva del recurso a la guerra o la revolución, de modo que las disputas entre los miembros de la sociedad pueden ser resueltas en función del ideal de Justicia universal y homogéneamente compartido.

\section{LA HISTORICIDAD DE LA JUSTICIA}

Una de las cuestiones que queda indudablemente por resolver es la de la formación de un ideal de justicia universalmente compartido. Esta idea o ideal de justicia (o este «sentido de la justicia») no se puede dar por supuesto como existiendo por sí mismo en un Estado universal y homogéneo. Le falta una garantía última, un fundamento. Se nos plantea, pues, una cuestión filosófica de gran calado. KoJÈve se apresta a solucionarla mediante el esquema hegeliano de su Introduction à la lecture de Hegel; más concretamente, sirviéndose de la mencionada lectura del cuarto capítulo, sección A, de la Fenomenología del espíritu de HEGEL. No es el presente artículo el lugar ade-

23 «En tant qu'entité politique, l'État tend à se propager par la conquête, il essaye d'absorber purement et simplement les États étrangers. Mais en tant qu'entité juridique l'État se contente d'imposer à l'étranger son Droit interne. Autrement dit, il tend à créer une Fédération d'États ou un État fédéral, en devenant lui-même un des États fédérés, la Fédération ayant pour base et pour résultat l'existence d'un Droit unique, commun à tous les États fédérés, et impliquant —dans son aspecte de "Droit Public" - un élément de "Droit fédéral", réglant les rapports des États fédérés entre eux, en particulier l'organisation fédérale de la justice. Si la Fédération n'est pas universelle, si elle a des États-ennemis à l'extérieur, elle devra s'organiser elle-même en État proprement dit (fédéral). Ses éléments intégrants - les États fédérés-auront eux aussi des ennemis; ils seront donc des États. Mais ils auront toujours des ennemis communs et ne pourront se réconcilier avec eux qu'en commun: ils seront donc non pas des États souverains, mais des États fédérés. Seulement la Fédération aura tendance à se propager le plus possible. À la limite elle englobera l'ensemble de l'bumanité. Alors elle cessera d'être un État au sens propre du mot, n'ayant plus d'ennemi au-dehors. Et les États fédérés cesseront par suite, eux aussi, d'être des États véritables. La Fédération deviendra alors une simple Union juridique mondiale (du moins dans son aspect juridique, qui n'est pas le seul)». 
cuado para entrar en el asunto de si la lectura hegeliana que KojÈve lleva a cabo es demasiado unilateral o de si, por el contrario, interpreta del mejor modo «lo que Hegel quiso decir». A juzgar por alguna alusión que le dedica, su propia filosofía residiría justamente en su peculiar interpretación de HEGEL ${ }^{24}$. Por ello nos parece del mayor interés la recuperación del Esquisse d'une phénoménologie du droit considerando que, según lo dicho más arriba, el autor habla en esa obra con voz propia, sin escudarse en el comentario de otra «autoridad» (a saber, HEGEL o lo que es más, HEGEL reelaborado con ingredientes marxistas y heideggerianos).

La base para la formación de la idea o ideal de justicia la pone la lucha antropogénica por el reconocimiento, esto es, la constitución de la humanidad a partir del afán de cada sujeto por ser reconocido por el otro. Huelga el insistir en el sumo interés de averiguar cómo se ha formado la idea o ideal de justicia, teniendo en cuenta que el contenido del derecho depende de tal idea. Pues bien, la respuesta empieza por abordar la lucha a muerte en aras del reconocimiento entre dos sujetos que arriesgan su propia vida a fin de ser reconocidos por el otro «en una franja de la ultrahistoria». En principio, las figuras hegelianas de la libertad y la dependencia de la conciencia (amo y esclavo) no responden a un hecho histórico fechable cronológicamente. Son más bien el resultado de un modelo «fenomenológico» (Fenomenología del Espíritu) de las relaciones entre los hombres como seres conscientes que luchan por el reconocimiento. En el híbrido filosófico, si se nos permite la expresión, de HeGEL y KoJÈve, se trata del desarrollo mismo de la historia de la humanidad.

Nos hallamos ahora mismo en la segunda sección del Esquisse d'une phénoménologie du droit, enfrentados a una lucha entre dos consciencias que pretenden el reconocimiento mutuo, y esta lucha afecta a la mera supervivencia animal. Cierto que, en busca del humano que está por acontecer, el animal se verá trascendido. Solo que en la lucha habrá un vencedor, el amo, y un perdedor, el esclavo. En términos de la idea o ideal de la justicia, ocurre que habrá una concepción de la justicia propia del amo reconocido como tal y una concepción de la justicia del esclavo. A la concepción de la justicia del amo la llamará KOJĖVE «justicia como igualdad» y, a la concepción de la justicia del esclavo, «justicia como equivalencia».

La justicia como igualdad surge de la perspectiva del amo, del sujeto vencedor en la lucha por el reconocimiento. Es una justicia de estatus. El amo solo reconocerá como iguales a otros amos, esto es, a aquellos que también han visto reconocida su humanidad por haber arriesgado su vida, negándola y trascendiéndola con vistas a un fin propiamente humano. La justicia de la igualdad es, paradójicamente, una justicia aristocrática. Ello significa, claro está, que el esclavo no merece siquiera consideración: que es lo otro, no reconocido en absoluto, al servicio del amo y poseído por él (su propiedad). En rigor, no hay desigualdad entre el amo y el esclavo, al existir entre ambos una diferencia cualitativa esencial que excluye toda noción de desigualdad. La justicia aristocrática es una justicia de la igualdad, pero no se asemeja en nada a la equivalencia porque nada tiene que ver con los contratos o las relaciones entre individuos. Es justo, ni más ni menos, que quienes han arriesgado su vida por un ideal humano y han salido

24 KOJÈvE habría manifestado en más de una ocasión que su lectura y comentario de HEGEL era una «obra de propaganda política», por ejemplo en una carta al fenomenólogo vietnamita TRAN DuC THAO, y que tras su interpretación de HEGEL se escondía su propia filosofía (ESQUIROL, 2006: 141-142). 
victoriosos sean reconocidos como iguales. KoJÈve busca un ejemplo de esta igualdad en las antiguas sociedades aristocráticas, como Esparta, donde los miembros de la clase dominante se llamaban a sí mismos hómoioi, los iguales. Así, es justo que los amos, en cuanto iguales, se traten en régimen de igualdad.

La justicia de la equivalencia, por contra, nace de la perspectiva del esclavo. Siendo este, propiamente, el sujeto que se ha rendido en la lucha por el reconocimiento, es decir, el que ha preferido conservar su vida a afrontar la lucha a muerte con todas sus consecuencias, sucede que la condena a servir a un amo (del que reconoce la humanidad aun no siendo reconocido) equivale a gozar de la gracia por él concedida de poder mantenerse con vida. Se trata de un germen de contrato cuyos elementos dispares (conservación de la vida y servicio en régimen de esclavitud) son vistos como equivalentes de manera que cabe una transacción. Se trata, pues, de una justicia protoburguesa, dicho en términos kojevianos. La justicia, en tal caso, nada tiene que ver con la igualdad, sino con la compensación entre dos valores, y residirá justo en el intercambio de un valor - al principio de la lucha, la conservación de la vida animal todavía no humana - por otro valor — los servicios que el esclavo deberá rendir al amo-.

Esta equivalencia constituye la nueva idea de Justicia. Esta justicia de equivalencia se manifiesta en y por el consentimiento mutuo que pone fin a la Lucha entablada por un consentimiento mutuo que ponía de manifiesto la Justicia de igualdad. A la Justicia de la igualdad, se ha añadido así una Justicia de la equivalencia (KoJĖve, 1981: 255) ${ }^{25}$.

Reparemos en el hecho de que, pese a las enormes diferencias implicadas, la situación final en que desemboca la lucha primordial es justa desde un doble punto de vista. En primer lugar lo es porque los contendientes han iniciado la lucha de mutuo consentimiento; nadie ha obligado al otro a luchar, sino que se han batido por propia voluntad, desde la igualdad. Claro está que el resultado final es dispar, pero esta disparidad cualitativa entre amo y esclavo no puede ser vista como injusta por el amo ya que las condiciones iniciales eran perfectamente iguales. En segundo lugar, tampoco el esclavo, por su parte, ve propiamente injusticia en su situación de esclavitud; pero su percepción no obedece a la consciencia de una igualdad primera, sino a la consciencia de una equivalencia entre el conservar la vida (como seguridad) y el mantener una relación de trabajo para pagar (como servicio) la seguridad vital que, por así decirlo, le garantiza el amo. Por ello puede KOJÈvE afirmar que el hombre en la historia no es nunca uno, sino dos: amo y esclavo. No existen, por lo demás, sociedades puramente basadas en la justicia como igualdad o sociedades exclusivamente basadas en la justicia como equivalencia. Lo que existe siempre es un compromiso o combinación entre ambos ideales de la justicia, que sirven de referencia para la formación del derecho realmente existente en las sociedades. Así, pues, la idea o ideal de la Justicia que pone en práctica un $\mathrm{C}$ desinteresado e imparcial en el conflicto entre un $\mathrm{A}$ y un $\mathrm{B}$ en una sociedad histórica particular estará siempre inspirado en una mezcla entre los componentes de la justicia de la equivalencia y de la justicia de la igualdad, mezcla en la cual uno de los componentes tendrá prioridad sobre el otro.

25 «C'est cette équivalence qui constitue la nouvelle idée de Justice. Et c'est cette justice d'équivalence qui se manifeste dans et par le consentement mutuel qui met fin à la Lutte engagée par un consentement mutuel qui manifestait la Justice d'égalité. À la Justice égalitaire vient s'ajouter ainsi une Justice de l'équivalence». 
Se hace evidente que la justicia como igualdad (aristocrática y de estatus) se halla en relación antitética con la justicia como equidad (burguesa y de contrato). Esto es, la justicia del amo y la justicia del esclavo se mantienen en una oposición no resuelta. KOJÈvE acude de nuevo a HEGEL para explicar que la síntesis entre la justicia aristocrática y la justicia de raigambre burguesa no es más que la reconciliación de estas dos instancias en la justicia absoluta o del ciudadano, que no es ni la justicia de la igualdad ni la justicia de la equivalencia, sino que es la justicia de la equidad. La justicia como equidad representa la combinación perfecta entre las dos figuras de la justicia en la historia (la oposición histórica entre amo y esclavo), y lógicamente solo podrá darse con el fin de la historia, como resolución final de la tensión existente entre los dos ideales en pugna. En suma, para KoJÈve la ciudadanía, en cuanto superación de la antítesis entre amo y esclavo, implica el fin del proceso histórico o, lo que viene a ser lo mismo, este desemboca en la realización de la humanidad como ciudadanía, algo que solo puede tener lugar en el Estado universal y homogéneo.

Para explicar el funcionamiento del principio de la equidad en la justicia, KoJÈvE se sirve de un ejemplo que ilustra a la perfección cómo se unen en un solo criterio los elementos de la igualdad y la equivalencia:

Tomemos el ejemplo del reparto de alimentos para la comida. El principio de igualdad exigirá una distribución a partes iguales entre los titulares de derechos, y no se ocupará de nada más. Pero el principio de equivalencia se preguntará si las partes iguales son verdaderamente equivalentes. Si se comprueba que algunos están más hambrientos que otros, entonces no será así en absoluto. Se distribuirá pues de otra manera, haciendo partes proporcionales a la necesidad de alimento de cada cual. Una vez satisfecho el principio de equivalencia, ya no se irá más lejos. Pero el otro principio se verá lesionado por la desigualdad del reparto y tratará de eliminarla. Solo que, para no lesionar el principio de equivalencia, hará falta eliminar la desigualdad de los participantes. Luego se preguntará por qué unos están más hambrientos que otros. Y si se comprueba que esta diferencia se debe a que unos han desayunado y otros no, se velará por que, en adelante, todos puedan desayunar. El principio de equivalencia, por tanto, habrá impulsado una realización más perfecta del principio de igualdad, llevando a que el principio de igualdad se realice con mayor perfección. $\mathrm{Y}$ al volverse perfecta, la igualdad coincide con la equivalencia. Pues si los poseedores de derechos son realmente iguales, la igualdad de sus partes ya no diferirá de su equivalencia y su equivalencia no será sino su igualdad (KoJÈVE, 1981:315) ${ }^{26}$.

En el planteamiento expuesto, la equidad implica una transformación de la igualdad desde la equivalencia y una transformación de la equivalencia desde la igualdad. Esta síntesis conlleva una modificación de las condiciones de partida de forma que la igualdad se torna equivalente y la equivalencia igualitaria. Con dicha modificación se

26 «Reprenons l'exemple du partage d'une nourriture pour le dîner. Le principe d'égalité exigera un partage en parts égales entre les ayants droit, et il ne se préoccupera plus de rien. Mais le principe de l'équivalence se demandera si les parts égales son vraiment équivalentes. Si l'on constate que les uns ont plus faim que les autres, on verra qu'il n'est est rien. On partagera alors autrement, rendant les parts proportionnelles au besoin de la nourriture de chacun. Le principe étant ainsi satisfait on s'en tiendra là. Mais l'autre principe sera choqué par l'inégalité du partage et il essayera de l'éliminer. Seulement, pour ne pas choquer le principe de l'équivalence il faudra éliminer l'inégalité des participants. On se demandera donc pourquoi les uns ont plus faim que les autres. Et si l'on constate que cette différence résulte du fait que les uns ont déjeuné et les autres non, on veillera à ce que dorénavant tous puissent déjeuner. Le principe de l'équivalence aura donc incité celui de l'égalité à se réaliser plus parfaitement. Et en devenant parfaite l'égalité coïncide avec l'équivalence. Car si les ayants droit son vraiment égaux, l'égalité de leurs parts ne diffère plus de leur équivalence, leur équivalence n'est autre chose que leur égalité». 
realiza propiamente la justicia ciudadana: universalización del derecho y homogeneización social a la par. Es por ello que fin de la historia, realización de la justicia en el derecho ciudadano y Estado universal y homogéneo coinciden en un mismo movimiento evolutivo. Según KoJÈve, este estado final aún no ha llegado, pero se están dando ya sus potencialidades en base a los principios revolucionarios de la libertad, la igualdad y la fraternidad. Como manifestó de forma claramente polémica en la Introduction à la lecture de Hegel, desde la batalla de Jena (1806) los cimientos del Estado universal y homogéneo ya estarían asentados. La expansión del código civil napoleónico equivaldría, pues, a la expansión del estadio final de la civilización, y su globalización consistiría nada menos que en la actualización de lo que ya estaría en potencia desde la Revolución Francesa (Robespierre) y la batalla de Jena (Napoleón) (KoJève, 1968: 436).

\section{CONCLUSIONES}

La filosofía del derecho de KoJève es un ímprobo esfuerzo con vistas a dotar de fundamento histórico la realización de la justicia. Vuelve para ello sobre la filosofía hegeliana partiendo de su lectura en términos marxistas, de forma que no concibe el derecho como algo estático, sino en permanente transformación histórica. Ahora bien, el criterio de materialización de la justicia o, lo que viene a ser lo mismo, de la realización del derecho en cuanto Idea o concepto realizado (según HEGEL) es inmanente al proceso histórico enfocado a la luz de la dialéctica entre el amo y el esclavo, que es la clave hermenéutica de la Lucha y el Trabajo en la Historia. De este modo, la kojeviana filosofía del derecho es una aplicación de la filosofía del mismo autor contenida en la Introduction à la lecture de Hegel.

Reviste suma importancia su descripción fenomenológica del Derecho en cuanto intervención de un tercero, $\mathrm{C}$, en la interacción de A y B. Ese tercero o C deberá ser imparcial y desinteresado al aplicar la idea o el ideal de la justicia resultante del proceso histórico formulado hegelianamente. Se trata, pues, de una filosofía del derecho que oscila entre el iusnaturalismo y el positivismo jurídico. Del derecho natural conserva el principio de que no todas las formas de interacción que reciben el nombre de «derecho» lo son propiamente; de ahí su insistencia en esclarecer lingüística y fenomenológicamente a qué llamamos «derecho», operando dicha clarificación a tenor de los paradigmas de intervención de terceros como árbitros en los conflictos sociales. Habría, en consecuencia, un marco supralegal a la luz del cual se podría dirimir qué es y qué no es «derecho». Ahora bien, la diferencia (esta vez de origen aristotélico) entre el derecho en potencia o facultativo, que no es, por tanto, «derecho» en sentido propio, y el derecho en acto, esto es, irresistible y realmente eficaz, acerca esta filosofía al iuspositivismo. KOJĖVE retiene este doble aspecto aparentemente contradictorio en un movimiento dialéctico en el cual el criterio supralegal (iusnaturalista) y el criterio de la eficacia (positivista) se sintetizan de tal modo que solo el derecho positivo del Estado universal y homogéneo puede realizar los principios verdaderos y esenciales del derecho. En suma, la naturalización de la legalidad se corresponde con la positivización de la Justicia.

Puede decirse que el Derecho «positivo» del Estado universal y homogéneo realiza el Derecho «natural», que no es sino el Derecho como tal, es decir, la «esencia» del Derecho. 
En el Estado universal y homogéneo, la teoría «racionalista» del Derecho coincide así con la teoría «histórica o sociológica». En ello reside el propio fondo del hegelianismo o, si se prefiere, de la comprensión dialéctica de la historia (KoJÈve, 1981: 91)27.

Lo más valioso que podría aportarnos una recuperación de la filosofía del derecho kojeviana sería su concepción de lo jurídico en términos de pacificación. A nuestro juicio, constituye una alternativa superadora del concepto schmittiano de lo político. Quizá resida su grandeza en el ideal regulativo (KERVEGAN, 2007b) que inspira la existencia de un Estado universal y homogéneo (o «Imperio socialista», como también lo llama KOJÈve), y que es el término final de la lucha histórica del esclavo por emanciparse del amo. La enorme distancia que actualmente nos separa de tal desenlace no representa una objeción concluyente a la teoría de KoJÈve dado que, en la medida en que prosigamos en la historia, habrá la posibilidad de realizar el ideal de una paz mundial que sea justa, o de perseverar al menos para acercarnos asintóticamente a la misma. Luego, según dicha interpretación, KOJÈvE propondría no tanto un final definitivo de la historia como una línea hermenéutica apta para juzgar los progresos jurídico-políticos con respecto a una pacificación e internacionalización siempre en marcha de las relaciones jurídicas.

Debe destacarse que el federalismo es una pieza clave en semejante expansión de lo jurídico, puesto que neutraliza la primacía de lo político en el Derecho. O dicho de otro modo, a la luz del proyecto kojeviano de un Estado global (universal) e igualitario (homogéneo) desaparece el problema de la fundamentación política del derecho. En efecto, las relaciones federales fusionan las soberanías de los distintos Estados federados, con lo cual ya no puede hablarse de los mismos como «Estados». Y por idéntica razón, la soberanía en su concepto clásico también estaría llamada a desaparecer al «final de la historia», en cuanto actualización del Estado universal y homogéneo. Soberano, en términos schmittanos, es quien decide. Pues bien, en el Estado universal y homogéneo podría decidir cualquiera posicionado como el tercero C. Desde luego, la consumación del referido estadio final solo resulta concebible en el supuesto de una perfecta mezcla (síntesis) de la justicia de la igualdad con la justicia de la equivalencia, mezcla que produciría una justicia de la equidad. De esta suerte, en un marco teórico en que lo jurídico iría ganando cada vez más terreno a lo político, los conflictos políticos de soberanía se transformarían en conflictos residuales.

Tiende a considerarse la filosofía de la historia que da pie a las mencionadas tesis en filosofía del derecho como una lectura creativa —o versión muy original — de las posibles consecuencias teóricas de la filosofía hegeliana. Ciertamente el hegelianismo de KoJève es sui generis; capta, no obstante, un elemento esencial: la dialéctica histórica o sucesión racional de las diversas épocas que HEGEL entendió como el desarrollo del «espíritu» (Geist). Pese a todo, la concepción kojeviana de la historia debe a KANT mucho más de lo que cabe admitir a primera vista. Así lo plantea Bryan-Paul Frost: «Kojève está mucho más cerca de la idea de Kant de lograr la paz a través de una federación de repúblicas que de la afirmación de Hegel de que la guerra es necesaria

27 «Nous pouvons donc dire que le Droit "positif" de l'État universel et homogène réalise le Droit "naturel", qui n'est rien d'autre que le Droit en tant que tel, c'est à dire l'"essence" du Droit. Dans l'État universel et bomogène, la théorie "rationaliste" du Droit coïncide donc avec la théorie "bistorique ou sociologique". C'est là le fond même du bégélianisme, ou, si l'on préfère, de la comprébension dialectique de l'bistoire». 
para la salud ética de las naciones» (Frost, 1999: 614) ${ }^{28}$. Diríase que KoJÈvE adapta el instrumental hegeliano a la defensa del irenismo kantiano atendiendo a la expansión transnacional de las relaciones jurídicas ${ }^{29}$.

La evolución jurídica hacia el ideal cosmopolita pasa necesariamente por las grandes federaciones. El propio KoJĖVE habría ofrecido una descripción de estos grandes poderes federales en su «Esquisse d'une doctrine de la politique française» de 1945, texto que iba destinado, según AufFret (1990: 288), a Charles De Gaulle y que diseñaba el papel de Francia en un mundo multipolar:

Los Estados-nación aún todopoderosos en el siglo XIX dejan de ser realidades políticas, esto es, Estados en el pleno sentido del término, igual que dejaron de serlo las baronías, las ciudades y los arzobispados medievales. El Estado moderno, la realidad política actual, requiere bases más amplias que las representadas por las Naciones propiamente dichas. Para ser políticamente viable, el Estado moderno debe descansar en una vasta unión «imperial» de naciones afines. El Estado moderno solo es un verdadero Estado siendo un Imperio (KoJÈVE, 2003: 88) ${ }^{30}$.

Del Imperio regional al Imperio mundial (Estado universal y homogéneo) hay evidentemente un largo trecho. Pero la lucidez de KoJÈve y su sentido histórico resultan apabullantes a la luz de la construcción de la Unión Europea en clave federal y del advenimiento de los grandes poderes regionales del planeta. Desde luego, la fundamentación hegeliana de su obra puede ponerse en tela de juicio. Sin embargo, no es nada fácil ignorar sus conclusiones jurídico-políticas, máxime si se entienden en función de los acontecimientos geopolíticos que han marcado la historia humana desde el final de la Segunda Guerra Mundial hasta nuestros días. La filosofía del derecho de KojÈvE es un especulativo banco de pruebas para dar con un posible sentido de las relaciones jurídico-políticas internacionales en el marco de una globalización que no descuide el sueño cosmopolita de raíces ilustradas.

\section{BIBLIOGRAFIA}

AufFret, D., 1990: Alexandre Kojève: La philosophie, l'État, la fin de l'Histoire, Paris: Grasset. EsquiROL, J. M., 2006: «Alexandre Kojève, o com es distreu un hegelià al segle XX», en J. VeRGÉs (ed.), Cons i neocons: El referons filosòfic, Girona: Documenta.

28 «Kojève is much closer to Kant's idea about achieving peace through a federation of republics than to Hegel's claim that war is necessary for the ethical health of nations».

${ }_{29}$ Hay un cierto kantismo en esta posición, pero es de lamentar que KojÈve no parezca ahondar demasiado en ello, lo cual ya ha sido subrayado por FrOST (1999) y KERVEGAN (2007b). Ni siquiera en su libro sobre KANT (KoJĖVE, 1973) menciona KojÈVE los escritos políticos del filósofo de Königsberg. Aun así, es obvia la filiación kantiana del constructo de la «unión jurídica mundial» en el Esquisse, alejado de la teoría del derecho internacional de Hegel ( $v . g r$. $\$ 331$ de la Rechtsphilosophie). KANT, al final de la sección segunda de la Paz perpetua, por citar un paso señalado, habla del «Derecho de ciudadanía mundial» como de algo más que una «fantasía jurídica». Será necesario profundizar más adelante, en otra ocasión, en este aspecto kantiano de la filosofía del derecho kojeviana.

30 «Les États-nations tout-puissants encore au XIXe siècle, cessent d'être des réalités politiques, des États au sens fort du mot, tout comme cessaient d'être des États les baronnies, les villes et les archevêchés médiévaux. L'État moderne, la réalité politique actuelle, exigent des bases plus larges que celles que représentent les Nations proprement dites. Pour être politiquement viable, l'État moderne doit reposer sur une vaste union "impériale" de nations apparentées. L'État moderne n'est vraiment un État que s'il est un Empire». 
Frost, B.-P., 1999: «A critical introduction to Alexandre Kojève's Esquisse d'une phénoménologie du droit», The Review of Metaphysics (52), 3: 595-640.

Hegel, G. W. F., 1993: Fundamentos de la filosofía del derecho, Madrid: Prodhufi.

- 2010: Fenomenología del espiritu, Madrid: Abada Editores.

Howse, R., 2006: «Europe and the New World Order: Lessons from Alexandre Kojève's engagement with Schmitt's “Nomos der Erde”», en Leiden Journal of International Law, 19.

Howse, R., y Frost, B.-P., 2007: «The Plausibility of the Universal and Homogeneous State», en A. KoJÈve, Outline of a Phenomenology of Right; translated, with and introductory essay and notes, by Bryan-Paul Frost and Robert Howse, edited by Bryan-Paul Frost, Lanham: Rowman \& Littlefield Publishers.

Jarczyc, G., y Labarrière, P.-J., 1996: De Kojève à Hegel. 150 ans de pensée hegelienne en France, Paris: Albin Michel.

KANT, I., 2003: Sobre la paz perpetua, Madrid: Tecnos.

Kervegan, J.-F., 2007a: Hegel, Carl Schmitt. Lo politico: entre especulación y positividad, Madrid: Escolar y Mayo.

— 2007b: «Kojève. Le temps du sage», en Hommage à Alexandre Kojève. Actes de la «Journée A. Kojève» du 28 janvier 2003, Paris: BnF, 16-27.

KoJÈve, A., [1947] 1968: Introduction à la lecture de Hegel, Paris: Gallimard.

- 1973: Kant, Paris: Gallimard.

- 1981: Esquisse d'une phénoménologie du droit, Paris: Gallimard.

- 2003: «Esquisse d'une doctrine de la politique française», Hommage à Alexandre Kojève. Actes de la «Journée A. Kojève» du 28 janvier 2003, Paris: BnF, 86-98.

Murphy, G., 2011: «Alexandre Kojève: Cosmpolitanism at the End of History», en L. TREPANIER y K. M. HABIB (eds.), Cosmopolitanism in the Age of Globalization: Citizens without States, Lexington: University Press of Kentucky.

Roth, M. S., 1983: «A note on Kojève's phenomenology of right», Political Theory (11), 3 : 447-450.

SChmitT, C., 1991: El concepto de lo político: texto de 1932 con un prólogo y tres corolarios, Madrid: Alianza.

TERRÉ, F., 2011: «La legitimidad de lo político», Anuario de Derechos Humanos (12), 1: 415-438. 